\title{
Multiwavelength diagnostics of accretion in an X-ray selected sample of CTTSs
}

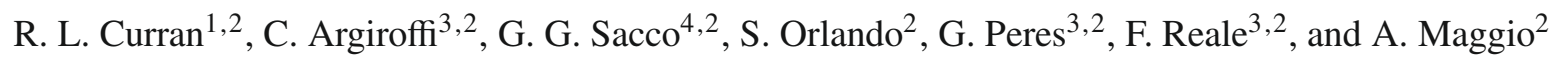 \\ 1 Present Address: Department of Physics, Rochester Institute of Technology, 84 Lomb Memorial Drive, Rochester, NY, 14623, USA \\ e-mail: rlcsps@rit.edu \\ 2 INAF - Osservatorio Astronomico di Palermo, Piazza del Parlamento 1, 90134 Palermo, Italy \\ 3 Dipartimento di Scienze Fisiche ed Astronomiche, Università degli Studi di Palermo, Piazza del Parlamento 1, 90134 Palermo, Italy \\ ${ }^{4}$ Chester F. Carlson Center for Imaging Science, Rochester Institute of Technology, 54 Lomb Memorial Drive, Rochester, NY 14623, \\ USA
}

Received 3 August 2010 / Accepted 18 November 2010

\begin{abstract}
Context. High resolution X-ray spectroscopy has revealed soft X-rays from high density plasma in classical T Tauri stars (CTTSs), probably arising from the accretion shock region. However, the mass accretion rates derived from the X-ray observations are consistently lower than those derived from UV/optical/NIR studies.

Aims. We aim to test the hypothesis that the high density soft X-ray emission originates from accretion by analysing, in a homogeneous manner, optical accretion indicators for an X-ray selected sample of CTTSs.

Methods. We analyse optical spectra of the X-ray selected sample of CTTSs and calculate the accretion rates based on measuring the $\mathrm{H} \alpha, \mathrm{H} \beta, \mathrm{H} \gamma, \mathrm{He}$ II $4686 \AA$, He I $5016 \AA$, He I $5876 \AA$, O I $6300 \AA$, and He I $6678 \AA$ equivalent widths. In addition, we also calculate the accretion rates based on the full width at $10 \%$ maximum of the $\mathrm{H} \alpha$ line. The different optical tracers of accretion are compared and discussed. The derived accretion rates are then compared to the accretion rates derived from the X-ray spectroscopy.

Results. We find that, for each CTTS in our sample, the different optical tracers predict mass-accretion rates that agree within the errors, albeit with a spread of $\approx 1$ order of magnitude. Typically, mass-accretion rates derived from $\mathrm{H} \alpha$ and He I $5876 \AA$ are larger than those derived from $\mathrm{H} \beta, \mathrm{H} \gamma$, and $\mathrm{O}$ I. In addition, the $\mathrm{H} \alpha$ full width at $10 \%$, whilst a good indicator of accretion, may not accurately measure the mass-accretion rate. When the optical mass-accretion rates are compared to the X-ray derived mass-accretion rates, we find that: a) the latter are always lower (but by varying amounts); b) the latter range within a factor of $\approx 2$ around $2 \times 10^{-10} M_{\odot} \mathrm{yr}^{-1}$, despite the former spanning a range of $\approx 3$ orders of magnitude. We suggest that the systematic underestimate of the X-ray derived mass-accretion rates could depend on the density distribution inside the accretion streams, where the densest part of the stream is not visible in the X-ray band because of the absorption by the stellar atmosphere. We also suggest that a non-negligible optical depth of X-ray emission lines produced by post-shock accreting plasma may explain the almost constant mass-accretion rates derived in $\mathrm{X}$-rays if the effect is larger in stars with higher optical mass-accretion rates.
\end{abstract}

Key words. accretion, accretion disks - circumstellar matter - stars: pre-main sequence - techniques: spectroscopic

\section{Introduction}

The process of stellar mass-accretion is an important aspect of star formation that is still not fully understood. Not only is accretion responsible for building up the young star to its final mass, but it is also responsible for powering the mass outflows observed in star forming systems, which in turn remove the excess angular momentum, and prevent the star from undergoing spin-up. In addition, understanding the mass-accretion rate will significantly enhance our understanding of the inner disk, disk evolution, and the eventual formation of planets. Stellar accretion can be briefly summarised as follows: material passes from the envelope through the accretion disk - which is truncated at a radius $R_{\text {in }}$ by the strong magnetic field of the central star. In this inner region of the disk, the material then flows along the star-disk magnetic-field lines (flux tubes) - at $\sim$ free-fall velocity - onto the central star, where a strong shock is formed as the accreting material impacts onto the stellar surface.

UV veiling, $\mathrm{H} \alpha, \mathrm{Pa} \beta, \mathrm{Br} \gamma$, O I, Ca II, and $\mathrm{He} \mathrm{I}$ emission lines have all previously been used to probe the accretion scenario in young stars and brown dwarfs (e.g. Natta et al. 2004, 2006;
Mohanty et al. 2003, 2005). These studies have been based on the hypothesis that the UV veiling arises from the hot shock region (e.g. Calvet \& Gullbring 1998), whereas the broad permitted emission lines emitted from CTTSs are found in the infalling magnetospheric flow (Muzerolle et al. 1998). The Ca II lines have been found to be particularly effective infall tracers (Muzerolle et al. 1998; Mohanty et al. 2005), their flux being strongly correlated with the accretion rate, although other lines such as $\mathrm{H} \alpha$ and He I $5876 \AA$ also display strong correlations with the accretion rate (Herczeg \& Hillenbrand 2008).

For the past few decades, it has been known that premain-sequence stars have strong X-ray emission (Feigelson \& DeCampli 1981). This X-ray emission was thought to have the same origin as that of main-sequence stars: low-density plasma $\left(n_{\mathrm{e}} \sim 10^{10} \mathrm{~cm}^{-3}\right.$ enclosed in coronal loop structures and heated to temperatures of $T \sim 10^{6}-10^{7} \mathrm{~K}$ (Feigelson \& Montmerle 1999). High resolution X-ray spectroscopy has detected soft $(E<0.7 \mathrm{keV}) \mathrm{X}$-ray emission, originating in high density $(n>$ $10^{11} \mathrm{~cm}^{-3}$ ) plasma of temperatures $\sim 3 \mathrm{MK}$ (see Telleschi et al. 2007, and references therein). This soft X-ray emission has been proposed to be caused by mass accretion. This interpretation is 
based on a simple model: assuming the accretion flow has a freefall velocity $v \sim 500 \mathrm{~km} \mathrm{~s}^{-1}$, it becomes heated by the shock (because of the impact of the accreting material with the stellar surface) at a temperature of $T \sim(3 / 16)\left(\mu m_{\mathrm{H}} v^{2}\right) \sim 3 \times 10^{6} \mathrm{~K}$, and then cools radiatively (Gullbring 1994; Calvet \& Gullbring 1998; Lamzin 1998; Gunther et al. 2007; Brickhouse et al. 2010) producing strong X-ray emission.

Supporting this interpretation is the fact that this plasma component i) has never been observed in non-accreting stars, and ii) is too dense to be of coronal origin. This interpretation is also supported by time-dependent models of radiative accretion shocks in CTTSs (Koldoba et al. 2008; Sacco et al. 2008, 2010; Orlando et al. 2010). In particular, Sacco et al. (2008) carried out a detailed hydrodynamic modeling of the interaction between the accretion flow and the stellar chromosphere, synthesizing the high resolution X-ray spectrum, as it would be observed with the Reflection Grating Spectrometers (RGS) on board the XMMNewton satellite. They found excellent agreement between the predicted and observed X-ray spectra, supporting once again the idea that this X-ray emission originates mainly from accretion shocks.

However, there are observational results that are difficult to reconcile with this framework. In particular, the mass-accretion rates derived from X-rays are usually systematically lower than the accretion rates derived from optical and UV data. As an example, for TW Hya, X-rays indicate that $\dot{M} \sim 1 \times 10^{-11} M_{\odot} \mathrm{yr}^{-1}$ (Stelzer et al. 2004), while $\mathrm{H} \alpha$ and UV find $5 \times 10^{-10} M_{\odot} \mathrm{yr}^{-1}$ (Muzerolle et al. 2000). The same disagreement is found for BP Tau: $\dot{M} \sim 9 \times 10^{-10} M_{\odot} \mathrm{yr}^{-1}$ from X-rays (Schmitt et al. 2005), and $3 \times 10^{-8} M_{\odot} \mathrm{yr}^{-1}$ from UV (Gullbring et al. 1998). To date, only three of these comparisons have been made in the literature (TW Hya; BP Tau; MP Mus, Argiroffi et al 2009), and each has used different UV/optical accretion measurements and differing means of calculating the $\mathrm{X}$-ray accretion rate. To confirm that the soft X-ray emission arises from the accretion process, and understand the discrepancies between the optical and $\mathrm{X}$-ray accretion rates, we have homogeneously analysed optical data for all the CTTSs for which high-resolution X-ray spectra, of good $\mathrm{S} / \mathrm{N}$ in the $[0.5,1.0] \mathrm{keV}$ range, have been gathered. We compare the X-ray derived mass-accretion rates to those measured from the $\mathrm{H} \beta$, He I $5876 \AA$, O I $6300 \AA$, H $\alpha$ equivalent width and $\mathrm{H} \alpha$ full-width at $10 \%$.

The structure of the paper is as follows. In Sect. 2, we present our sample of CTTSs previously observed with Chandra and XMM. We briefly discuss the parameters of each CTTS (summarised in Table 1), along with the previously published X-ray data. In Sect. 3, we discuss the data reduction and analysis of the optical data (Sect. 3.1), and the derivation of mass-accretion rates from the X-ray data (Sect. 3.2). In Sect. 4.1, we draw comparisons about the different optical tracers of accretion and discuss the mass-accretion rates derived from these tracers. In Sect. 4.2, we analyse and discuss the variability of TW Hya. In Sect. 4.3, we compare the optical and X-ray mass-accretion rates and discuss possible scenarios to explain our results, and in Sect. 5 we present our conclusions.

\section{The sample}

The sample consists of all the CTTSs currently observed with high-resolution X-ray spectroscopy (either with Chandra, or RGS on XMM-Newton) and for which O VII triplet lines have been measured, and which also have high resolution optical echelle spectroscopy available in the data archives. The X-ray data have previously been published, with the published fluxes of
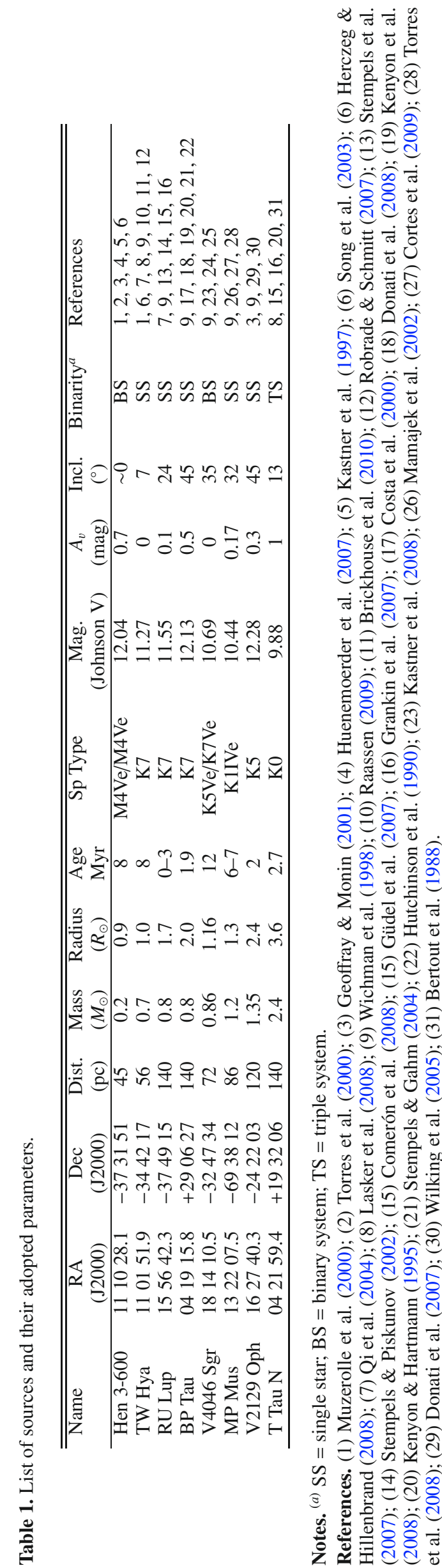
Table 2. Optical archival data information for our sample of CTTSs.

\begin{tabular}{|c|c|c|c|c|}
\hline Name & Telescope & Instrument & $\begin{array}{c}\text { Observation Date } \\
\text { (yyyymmdd) }\end{array}$ & $\begin{array}{c}\text { Exposure time } \\
\text { (s) }\end{array}$ \\
\hline Hen 3-600 & ESO $2.2 \mathrm{~m}$ & FEROS & 20040512 & 900 \\
\hline TW Hya & $\mathrm{ESO} 2.2 \mathrm{~m}$ & FEROS & 20070426 & 900 \\
\hline RU Lup & VLT & UVES & 20050814 & 900 \\
\hline BP Tau & TNG & SARG & 20071220 & 3600 \\
\hline V4046 Sgr & VLT & UVES & 20050815 & 900 \\
\hline MP Mus & $\mathrm{ESO} 2.2 \mathrm{~m}$ & FEROS & 20060418 & 3000 \\
\hline V2129 Oph & VLT & UVES & 20020417 & 900 \\
\hline T Tau & TNG & SARG & 20061130 & 3600 \\
\hline
\end{tabular}

the $\mathrm{O}$ VII used to derive the mass-accretion rate for each star. The parameters for each source, used in our calculations are given in Table 1. Below are brief descriptions of each source.

Hen 3-600 is a multiple star system and a member of the TW Hydra Association, at a distance of 45 pc. This is an average distance taken from Huenemoerder et al. (2007), who used the photometric distance from Kastner et al. (1997) and the typical distance adopted for stars belonging to the TW Hya association. The primary components (A and $\mathrm{B}$ ) of this system are separated by 1."4, and component $\mathrm{A}$ is surrounded by a dusty disc (Jayawardhana et al. 1999). Component A is also inferred (by Huenemoerder et al. 2007) to be almost pole-on, on the basis of its large mid-IR excess and a negligible optical reddening (based on a measured $B-V=1.52$, which is nearly that of an unreddened M3 photosphere; Johnson 1966). Huenemoerder et al. (2007) discuss the X-ray Chandra observations of Hen 3600 , and find high density plasma, and a larger "soft excess" for component A, than B.

TW Hya is one of the closest known CTTSs, at a distance of only $\sim 56 \mathrm{pc}$ (Wichmann et al. 1998). It has a mass of $0.7 M_{\odot}$, a radius of $1 R_{\odot}$ (Muzerolle et al. 2000), and is orientated such that it is seen nearly pole-on (Kastner et al. 1997). Previous X-ray data have been published by Kastner et al. (2002), Raassen (2009), Brickhouse et al. (2010), and Stelzer \& Schmitt (2004), who found a strong soft X-ray emission produced by high density plasma.

$R U$ Lup is located at a distance of 140 pc (Hughes et al. 1993). It has a mass and radius of $0.8 M_{\odot}$ and $1.7 R_{\odot}$, respectively (Stempels \& Piskunov 2002), it is hardly affected by absorption (Herczeg et al. 2005) and is believed to be viewed almost pole-on (Stempels \& Piskunov 2002). XMM data are discussed by Robrade \& Schmitt (2007), who find cool, high density plasma that they conclude is indicative of an accretion shock origin.

BP Tau is a CTTS in the Taurus-Auriga molecular cloud at a distance of $140 \mathrm{pc}$ (Kenyon et al. 2008). It has a spectral type of $\mathrm{K} 7$, and a mass of $0.8 M_{\odot}$ (Kenyon \& Hartmann 1995; Güdel et al. 2007). The XMM-Newton spectra were previously discussed by Schmitt et al. (2005) and Robrade \& Schmitt (2006), who reported high density soft X-ray emission from the $\mathrm{O}$ VII triplet, which might be due to accretion shocks.

V4046 Sgr is a nearby spectroscopic binary CTTS that is isolated from any dark cloud or molecular cloud, has negligible extinction, and exhibits evidence of a circumstellar disk (Hutchinson et al. 1990). The period is reliably determined to be 2.4213459 days (Stempels \& Gahm 2004) and the separation is estimated to be $\sim 10 R_{\odot}$ (Quast et al. 2000). The observed spectral energy IR distribution is consistent with the disk having an inner radius of $1.8 \mathrm{AU}$, thus the disk is circumbinary. The inclination of the system is $\sim 35-45^{\circ}$. Chandra data of V4046 Sgr is presented by Günther et al. (2006), who found that the X-ray emission is due to high density plasma.

MP Mus is a K1 IVe type star located in the lower Centaurus Crux (LCC) association at a distance of $\sim 86 \mathrm{pc}$. It is known to have a dusty disk (Mamajek et al. 2002; Silverstone et al. 2006) with a dust mass of $\sim 5 \times 10^{-5} M_{\odot}$ (Carpenter et al. 2005). Batalha et al. (1998) studied the variability in $B, V, R$ and $I$ bands, and found that it has low variability for a CTTS. The XMM data for MP Mus were previously published by Argiroffi et al. (2007), who found evidence of a high density plasma responsible for the soft X-ray emission.

V2129 Oph is the brightest CTTS in the $\rho$ Oph cloud, at a distance of 120 pc (Lombardi et al. 2008). It is a distant binary, with its very low-mass companion (possibly a brown dwarf) being about 50 times fainter than the CTTS in the $V$ band (Geoffray \& Monin 2001). The CTTS is inferred to have a mass of $1.35 M_{\odot}$ and a radius of $2.4 R_{\odot}$. Chandra data have recently been obtained (Argiroffi et al., in prep.) but not yet published. We include the optical analysis in this paper to allow an easy comparison once the X-ray data is published.

$T$ Tau $N$ is another CTTS located in Taurus-Auriga. It is an optically visible part of a triple system, along with T Tau Sa and $\mathrm{Sb}$, which are its "infrared companions". It has a spectral type of K0 (Kenyon \& Hartmann 1995) and a mass of $2.7 M_{\odot}$ (Güdel et al. 2007). It is oriented such that it is seen almost pole-on (Solf \& Böhm 1999; Akeson et al. 1998). X-ray data from XMM and Chandra were discussed in Güdel et al. (2007) who found a "soft excess" but no evidence of high density plasma.

\section{Data analysis}

\subsection{Optical data analysis}

We retrieved high-resolution echelle spectra spanning the optical wavelength range from the archives. No single instrument had observed the entire sample (partly because of their locations). Data from three instruments, FEROS on the $2.2 \mathrm{~m}$ ESO telescope in Chile, UVES on the VLT in Chile, and SARG on the TNG in La Palma were used in this analysis. Details of the telescope, instrument, observation date, and exposure times for each source can be found in Table 2. Relevant calibration files were downloaded with each dataset and used in the data reduction process.

The data from the three instruments were reduced in the standard manner using IRAF routines to de-bias, flat-field, and extract the spectra. Wavelength calibrations were carried out based on observations of ThArNe arcs for FEROS, ThAr arcs for UVES, and thorium arcs for SARG.

Line luminosities, $L_{\text {line }}$, were calculated from the equivalent width of each emission line (see Table 3 ). The continuum fluxes were estimated from the $V$ magnitudes of the stars, following a method similar to that used by Mohanty et al. (2005) and Dahm (2008). The continuum flux at $5500 \AA$ was calculated using the extinction-corrected observed $V$ magnitude, the distance, and the photometric zero point of the Johnson $V$ filter (at the central wavelength $5500 \AA$ ) of $3.75 \times 10^{-9} \mathrm{erg} \mathrm{cm}^{-2} \mathrm{~s}^{-1} \AA^{-1}$ (Mitchell \& Johnson 1969). The continuum flux at the wavelength of each of the analysed emission lines also required correction for the stellar spectral response - which was measured using the Pickles Spectrophotometric Atlas of Standard Stellar Spectra (Pickles 1985) in conjunction with the observed stellar spectral type. It is worth noting that using the observed $V$ magnitude we take into account the continuum excess due to the accretion process. 
Table 3. Measured equivalent widths for the accretion $\operatorname{tracers}^{a}$, along with the measured full width at $10 \%$ for the $\mathrm{H} \alpha$ line.

\begin{tabular}{lccccccccc}
\hline \hline Source & H $\gamma$ 4340 $\AA$ & He II $4686 \AA$ & H $\beta$ 4861 $\AA$ & He I 5016 $\AA$ & He I 5876 $\AA$ & O I 6300 & H $\alpha 6563 \AA$ & H $\alpha$ FW $10 \%$ & He I 6678 $\AA$ \\
& $\AA$ & $\AA$ & $\AA$ & $\AA$ & $\AA$ & $\AA$ & $\AA$ & $\mathrm{km} \mathrm{s}^{-1}$ & $\AA$ \\
\hline Hen 3-600 & -7.51 & $\ldots$ & -7.93 & -0.32 & -0.96 & -0.37 & -21.81 & 282.29 & -0.37 \\
TW Hya & -18.49 & -0.48 & -31.9 & -0.32 & -3.16 & -0.54 & -141.58 & 407.55 & -0.7 \\
RU Lup & NA & NA & -28.34 & -9.25 & -4.55 & -1.17 & -68.95 & 578.42 & -1.88 \\
BP Tau & NA & -0.6 & -19.82 & -0.27 & -1.08 & -0.37 & -58.63 & 413.41 & -0.31 \\
V4046 Sgr & NA & NA & -5.97 & $\ldots$ & -0.41 & -0.12 & -32.66 & 515.19 & $\ldots$ \\
MP Mus & -1.34 & $\ldots$ & -3.16 & $\ldots$ & -0.3 & -0.23 & -23.47 & 502.9 & 0.16 \\
V2129 Oph & NA & NA & -2.54 & $\ldots$ & -0.33 & -0.13 & -12.07 & 274.24 & -0.04 \\
T Tau & NA & -0.1 & -12.05 & -0.6 & -0.55 & -0.7 & -42.45 & 419.71 & 0.08 \\
\hline
\end{tabular}

Notes. ${ }^{(a)}$ Negative values represent emission. Note some sources show lines in absorption. There are no measurements where the spectra show no indication of a line being present. NA indicates where the wavelength of the line was not covered in the observation.

Table 4. Coefficients used in Eq. (1), for each emission line, from Herczeg \& Hillenbrand (2008).

\begin{tabular}{lccc}
\hline \hline Line & $\begin{array}{c}\text { Wavelength } \\
(\AA)\end{array}$ & $a$ & $b$ \\
\hline $\mathrm{H} \gamma$ & 4349 & $3.0 \pm 0.2$ & $1.24 \pm 0.04$ \\
$\mathrm{He}$ II & 4686 & $3.7 \pm 0.5$ & $1.04 \pm 0.08$ \\
$\mathrm{H} \beta$ & 4861 & $2.6 \pm 0.2$ & $1.22 \pm 0.05$ \\
$\mathrm{He}$ I & 5016 & $3.3 \pm 0.3$ & $1.04 \pm 0.05$ \\
$\mathrm{He}$ I & 5876 & $5.3 \pm 0.7$ & $1.46 \pm 0.12$ \\
$\mathrm{O}$ I & 6300 & $2.8 \pm 0.8$ & $0.96 \pm 0.16$ \\
$\mathrm{H} \alpha$ & 6563 & $2.0 \pm 0.4$ & $1.2 \pm 0.11$ \\
$\mathrm{He}$ I & 6678 & $5.2 \pm 0.8$ & $1.37 \pm 0.13$ \\
\hline
\end{tabular}

The mass-accretion rates were calculated following the empirical relation between accretion and line luminosities found and described by Herczeg \& Hillenbrand (2008)

$\log L_{\text {acc }}=a+b \log L_{\text {line }}$,

where the coefficients $a$ and $b$ (listed in Table 4) were calculated - by Herczeg \& Hillenbrand (2008) - by comparing optical emission-line fluxes and accretion luminosities measured from the UV continuum excess. Errors in the coefficients $a$ and $b$ depend on the scatter in the line luminosity values around the best-fit relation (from a large sample of sources), and therefore take into account several effects, such as optical depth and any contribution to the line emission from stellar outflows.

Once the accretion luminosity, $L_{\mathrm{acc}}$, was calculated, it was possible to calculate the mass-accretion rate via

$\dot{M}=\left(1-\frac{R_{*}}{R_{\text {in }}}\right)^{-1} L_{\mathrm{acc}} \frac{R_{*}}{G M_{*}} \approx 1.25 L_{\mathrm{acc}} \frac{R_{*}}{G M_{*}}$,

where $\left(1-R_{*} / R_{\text {in }}\right)^{-1} \approx 1.25$ is estimated by assuming the accreting gas falls onto the star from the truncation radius of the disk, $R_{\text {in }} \approx 5 R_{*}$ (Gullbring et al. 1998).

We also calculated the mass-accretion rate based on the $\mathrm{H} \alpha$ full width at $10 \%$, using the equation (Natta et al. 2004)

$\log \dot{M} \approx-12.9( \pm 0.3)+9.7( \pm 0.7) \times 10^{-3} \mathrm{H} \alpha 10 \%$,

where $\mathrm{H} \alpha 10 \%$ is the $\mathrm{H} \alpha 10 \%$ full width in $\mathrm{kms}^{-1}$ and $\dot{M}$ is in $M_{\odot} \mathrm{yr}^{-1}$. The derived mass-accretion rates, along with a (weighted) mean optical accretion rate, $\left\langle\dot{M}_{\text {Opt }}\right\rangle$, are listed in Table 5. The mean optical accretion rate was calculated using all the mass-accretion rates calculated via Eqs. (1) and (2) and therefore does not include the mass-accretion rate derived from the $\mathrm{H} \alpha 10 \%$ width (see Sect. 4.1 for more details).

\subsection{X-ray data analysis}

It is also possible to infer the mass-accretion rate $\dot{M}$ of CTTSs from their soft X-ray emission (e.g. Schmitt et al. 2005). This method for deriving $\dot{M}$ is based on the assumption that the whole soft X-ray emission is due to accreting material and not to coronal plasma, and that the post-shock zone can be described as a stationary isothermal slab of plasma at constant velocity and density. Hydrodynamical simulations (Sacco et al. 2008, 2010) demonstrated that, even if the post-shock zone is not stationary (and characterized by quasi-periodic shock oscillations), the time-averaged properties of the post-shock zone are, in general, well-described by the stationary model. Sacco et al. (2010) also demonstrated that the higher the post-shock temperature the larger the discrepancies between the stationary and the hydrodynamical models. This is mainly because the thermal conduction (which is not taken into account in the stationary model and is more efficient for higher post-shock temperatures) drains energy from the shock-heated plasma to the chromosphere through a thin transition region, and acts as an additional cooling mechanism (see also Orlando et al. 2010).

Where $n_{0}$ and $v_{0}$ represent the density and velocity of the pre-shock material, and $n_{1}$ and $v_{1}$ the corresponding post-shock values, the pre- and post-shock quantities are linked by the relations

$n_{1}=4 n_{0}, \quad v_{1}=\frac{1}{4} v_{0}, \quad T_{1}=\frac{3}{16} \frac{\mu m_{\mathrm{H}}}{k} v_{0}^{2}$,

where $T_{1}$ is the post-shock temperature, $\mu$ is the average mass per free particle $(\mu \approx 0.5)$, and we assume a strong shock regime. The volume occupied by the hot post-shock plasma is defined by the stream cross-section, $A$, and by the distance, $l$, covered by the plasma itself before cooling. Hence, where $\tau$ is the cooling time, $l$ is given by $v_{1} \tau$, and the emission measure $-E M-$ of the shock-heated plasma is $n_{1}^{2} A v_{1} \tau$. Therefore it is possible to derive the stream cross-section $A$, and the mass-accretion rate, from the $E M$ value measured from the soft $\mathrm{X}$-ray emission via

$\dot{M}=\mu m_{\mathrm{H}} n_{1} v_{1} A=\mu m_{\mathrm{H}} \frac{E M}{n_{1} \tau}=\mu m_{\mathrm{H}} \frac{E M P\left(T_{1}\right)}{3 k T_{1}}$,

where $\tau=3 k T /\left(n_{1} P(T)\right)$, with $P(T)$ indicating the plasma radiative losses per $E M$ unit. We used the above formula to obtain $\dot{M}$ from the X-ray data. In particular for each CTTS: 1) we evaluate $v_{0}$, hence $v_{1}$, assuming a free fall velocity from a distance of $5 R_{\star}$ (to correspond with the optical analysis); 2) we evaluate the post shock temperature $T_{1}$ from $v_{0}$ (see Eq. (4));3) the knowledge of 
$T_{1}$ allows us to derive the plasma $E M$ from the observed flux of the O VII resonance line located at $21.60 \AA$ via

$E M=\frac{L_{\mathrm{OVII}}}{G\left(T_{1}\right) A_{\mathrm{O}}}$,

where $G\left(T_{1}\right)$ is the emissivity function of the O VII resonance line at $21.60 \AA$, and $A_{\mathrm{O}}$ is the oxygen abundance.

We chose to base our $\dot{M}$ estimates on the flux of the O VII resonance line because this is mostly produced by plasma at $2 \mathrm{MK}$, which is the typical expected temperature for the plasma heated in the accretion shock, and because its flux measurement is available for almost all CTTSs observed with high resolution X-ray spectroscopy.

We note that this method allows us to derive $\dot{M}$ independently of knowledge about the plasma density $n_{1}$. On the other hand, this method depends on the plasma metallicity, which is needed to calculate the radiative losses $P\left(T_{1}\right)$ and link the $\mathrm{O}$ VII line flux to the corresponding $E M$. We note that X-ray observations provide us with calibrated spectra, making the line flux measurements a simple and solid procedure. This is particularly true for the O VII resonance line at $21.6 \AA$, which is an isolated line, hence whose flux estimate is unaffected by problems due to line blending or wrong continuum placement. We decided, therefore, to infer the $\dot{M}$ values, with the above method, starting from published values of the fluxes of the O VII resonance line.

In Table 6, we list, for each CTTS, the derived accretion rates and the main parameters used.

\section{Results and discussion}

\subsection{Comparison of optically derived accretion rates}

Table 5 shows the calculated mass-accretion rates for each star based on the different emission lines analysed. Although the sources were observed at different epochs, all the emission lines were observed simultaneously for each star. Therefore, one might naïvely expect the different tracers to lead to very similar mass accretion rates (if each line is tracing the same accretion phenomena). The top panel of Fig. 1 shows the different massaccretion rates calculated for each star in the sample (in order of increasing stellar mass). We find that all the different tracers agree within the errors, albeit with a spread of typically $\sim 1$ order of magnitude. We see no relation between mass-accretion rate and the mass of the stars. It should also be noted that there is no clear relation between the mass-accretion rate and the age of the stars (see Table 1). For example, V2129 Oph has an age of $2 \mathrm{Myr}$, whereas T Tau has an age of 2.7 Myr and their massaccretion rates are vastly different. In addition, V4046 Sgr has an age of $12 \mathrm{Myr}$ and MP Mus has an age of 6-7 Myr, and yet they have similar mass-accretion rates. The bottom panel of Fig. 1 shows the distribution of the difference between each mass-accretion rate estimate and $\left\langle\dot{M}_{\text {Opt }}\right\rangle$, the optical mean massaccretion rate. In general, $\mathrm{H} \alpha$ flux and He I $5876 \AA$ lead to higher mass-accretion rates than $\mathrm{H} \beta, \mathrm{H} \gamma$, and $\mathrm{O} \mathrm{I}$.

The measured $\mathrm{H} \alpha 10 \%$ mass-accretion rates are often at the extremes of the range of values found, but this is unsurprising given that the $\mathrm{H} \alpha$ line profiles are dependent on inclination, rotation effects (see Mohanty et al. 2005, for details), winds, and outflows. Figure 2 shows the $\mathrm{H} \alpha$ line profiles for our sample, some of which are very asymmetric. The $\mathrm{H} \alpha$ emission from Hen 3600 and MP Mus both have narrow peaks, but asymmetric broad wing emission. TW Hya, RU Lup, and BP Tau all have broader 


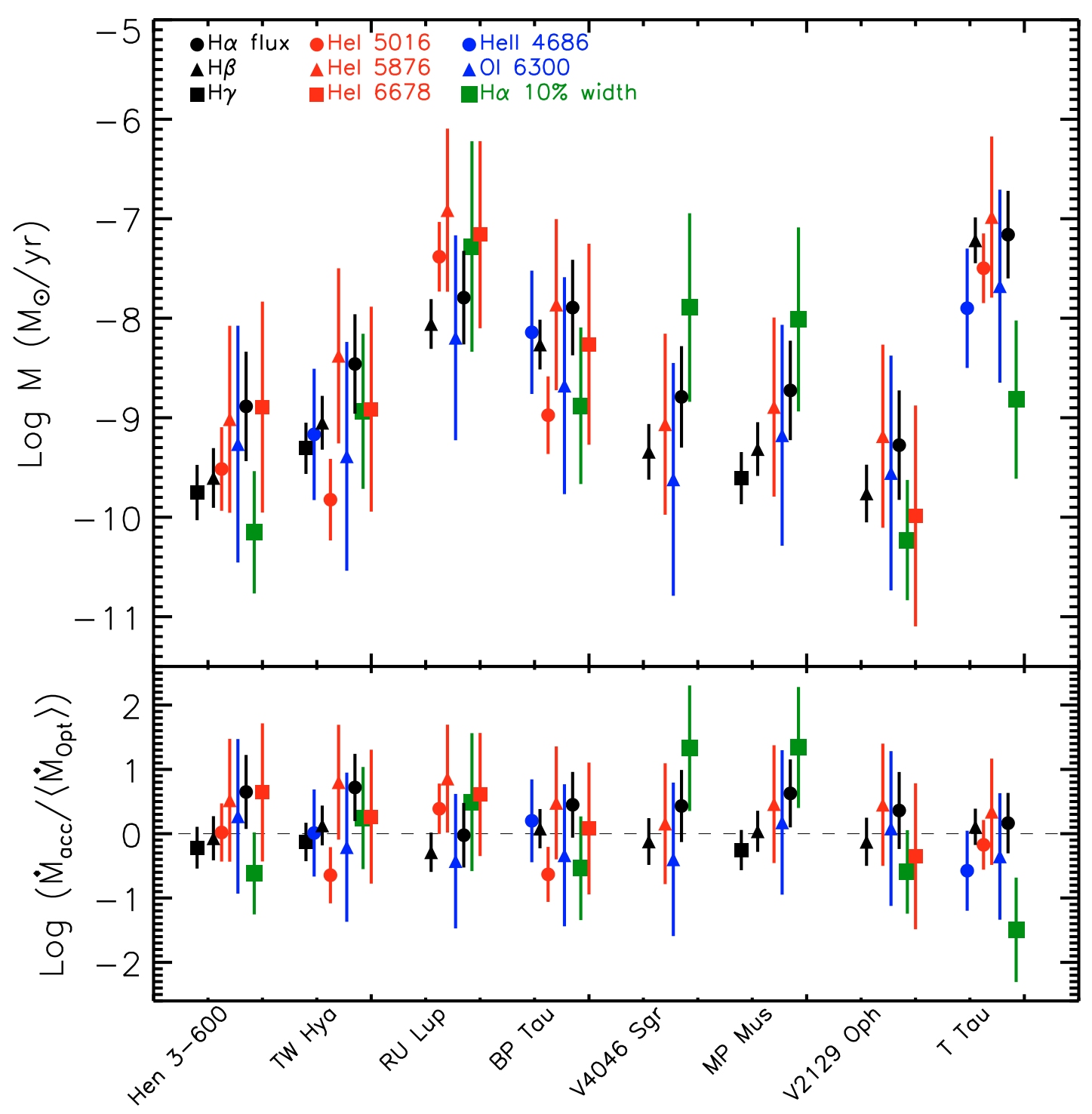

Fig. 1. Top panel: plot of the different mass-accretion rates from different accretion tracer emission lines, for each of the stars in the sample. The stars are in order of increasing mass, showing no relation between mass and mass-accretion rate. Bottom panel: plot showing the ratio of each mass accretion rate estimate to the optical mean of the accretion rate for each star. The different symbols/colors represent different accretion rate tracers (see upper left corner in the top panel).

peaked emission, albeit with some evidence of self-absorption. V4046 Sgr has an $\mathrm{H} \alpha$ line profile that has a broad peak and very broad wing emission. V2129 Oph and T Tau both show clear evidence of absorption in the $\mathrm{H} \alpha$ emission, with V2129 Oph having a very asymmetric profile with strong absorption of the blue-shifted wing/no blueshifted component. Interestingly, we see that the accretion rates derived from $\mathrm{H} \alpha 10 \%$ emission is at the lower part of the range of mass-accretion rates for Hen 3600, BP Tau, V2129 Oph, and T Tau, at the upper end of the range for RU Lup, V4046 Sgr and MP Mus, and in the middle of the range for TW Hya (see top panel of Fig. 1). The asymmetry of the emission line profile from V2129 Oph would clearly cause the mass-accretion rate of that object to be underestimated, but interestingly, the underestimation is not extreme, the difference between the $\mathrm{H} \alpha 10 \%$ mass-accretion rate and the optical mean mass-accretion rate being only $-1.7 \times 10^{-10} M_{\odot} \mathrm{yr}^{-1}$. For V4046 Sgr, MP Mus, and T Tau, these estimates are clear outliers from the rest of the accretion rate estimates (high estimates for the first two - as predicted from the line profiles, and lower for T Tau - which has a "normal" line profile, albeit with some absorption, but this does not affect the $10 \%$ intensity level). Since $\mathrm{H} \alpha 10 \%$ mass-accretion rate estimates are outliers for almost half our sample, and inclination, rotation, winds, and outflows may affect these mass accretion rates, we have excluded the $\mathrm{H} \alpha 10 \%$ mass-accretion rate estimates from the calculation of the optical mean mass-accretion rate listed in Table 5.

\subsection{Variability of TW Hya}

TW Hya is the only source within the sample to have been observed over a period of months (from March 2007 through to July 2007) on both short (nightly) and long (monthly) timescales, using the same instrumental set-up. We analysed 14 nights of data over a five month period for the purpose of studying the accretion variability. Our results are shown in Fig. 3. We find that the variability of a given tracer is small compared to the $\sim 1$ order of magnitude spread in mass-accretion rates from the different tracers. O I, $\mathrm{H} \alpha$, and He I $5876 \AA$ are the tracers with the least variability, whilst $\mathrm{H} \beta$ and $\mathrm{H} \gamma$ have the 

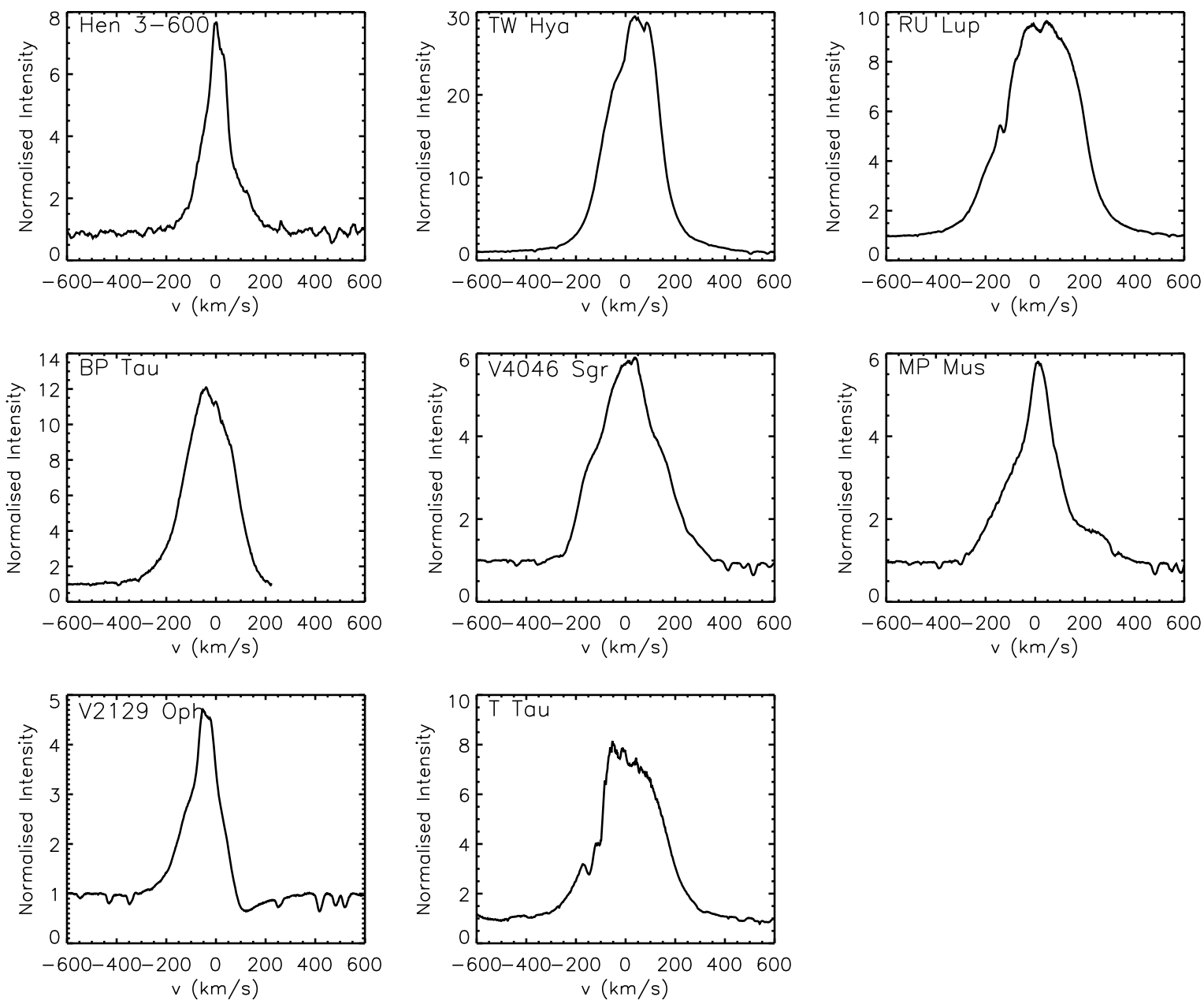

Fig. 2. The observed $\mathrm{H} \alpha$ line profiles for our sample. The data show many different line morphologies, with some very asymmetric lines e.g. V2129 Oph.

Table 6. Observed X-ray parameters and derived X-ray mass-accretion rates ${ }^{a}$.

\begin{tabular}{lccccccc}
\hline \hline Name & $\begin{array}{c}T \\
(\mathrm{MK})\end{array}$ & $\mathrm{O}, \mathrm{Ne}, \mathrm{Fe}$ & $\begin{array}{c}P(T) \\
\left(10^{-23} \mathrm{~cm}^{3} \mathrm{erg} \mathrm{s}^{-1}\right)\end{array}$ & $\begin{array}{c}\log L_{\mathrm{OVII}} \\
\left(\mathrm{erg} \mathrm{s}^{-1}\right)\end{array}$ & $\begin{array}{c}\log E M \\
\left(\mathrm{~cm}^{-3}\right)\end{array}$ & $\begin{array}{c}\log \dot{M} \\
\left(M_{\odot} \mathrm{yr}^{-1}\right)\end{array}$ & $\begin{array}{c}\text { References for } L_{\mathrm{OVII}} \\
\text { and abundances }\end{array}$ \\
\hline Hen 3-600 & 1.0 & $0.4,1.2,0.2$ & 5.7 & $27.5 \pm 0.2$ & $52.7 \pm 0.2$ & $-9.53 \pm 0.26$ & 1 \\
TW Hya & 3.1 & $0.2,1.8,0.3$ & 2.2 & $28.9 \pm 0.1$ & $53.4 \pm 0.1$ & $-9.74 \pm 0.05$ & 2 \\
RU Lup & 2.1 & $0.6,1.4,1.1$ & 7.2 & $28.9 \pm 0.1$ & $52.8 \pm 0.1$ & $-9.67 \pm 0.13$ & 3 \\
BP Tau & 1.8 & $0.6,1.5,0.3$ & 4.7 & $28.9 \pm 0.1$ & $52.8 \pm 0.1$ & $-9.78 \pm 0.08$ & 4,5 \\
V4046 Sgr & 3.3 & $0.1,1.0,0.1$ & 1.6 & $28.5 \pm 0.1$ & $53.5 \pm 0.1$ & $-9.84 \pm 0.11$ & 6,7 \\
MP Mus & 4.1 & $0.1,0.4,0.1$ & 1.3 & $28.6 \pm 0.1$ & $53.8 \pm 0.1$ & $-9.70 \pm 0.06$ & 8 \\
V2129 Oph & $\ldots$ & $\ldots$ & $\ldots$ & $\ldots$ & $\ldots$ & $\ldots$ & $\ldots$ \\
T Tau & 2.9 & $0.4,0.8,0.3$ & 2.4 & $29.4 \pm 0.1$ & $53.6 \pm 0.1$ & $-9.47 \pm 0.11$ & 9,10 \\
\hline
\end{tabular}

Notes. ${ }^{(a)}$ Chandra data for V2129 Oph have recently been obtained (Argiroffi et al., in prep.) but not published yet.

References. (1) Huenemoerder et al. (2007); (2) Stelzer \& Schmitt (2004); (3) Robrade \& Schmitt (2007); (4) Schmitt et al. (2005); (5) Robrade \& Schmitt (2006); (6) Günther et al. (2006); (7) Argiroffi C., priv. commun.; (8) Argiroffi et al. (2009); (9) Güdel \& Telleschi (2007); (10) Güdel et al. (2007).

largest variability. It could be argued that this small variability is expected given that TW Hya is viewed almost pole-on, and as such it should be possible to observe all active accretion regions about the facing pole at all times, therefore eliminating large changes in mass-accretion rates as the hotspots rotate out of view. The variability in the mass-accretion rates are not necessarily caused only by the inclination angle of the star-disk system, but possibly also by time-dependent accretion rates. The small amount of variability ( $\sim 0.25$ order of magnitude) that we observe in our TW Hya data may be due to a time-dependent accretion rate.

Alencar \& Batalha (2002) studied the TW Hya accretion rate for $1.5 \mathrm{yrs}$, and found the mass-accretion rate to vary between $10^{-9}$ and $10^{-8} M_{\odot} \mathrm{yr}^{-1}$ using the NaD line profile. Whilst these results fall within the range of mass accretion rates we measured using different tracers, they are indicative of a larger variability. This discrepancy may be due to the method used to estimate the mass-accretion rate or the longer time period covered 


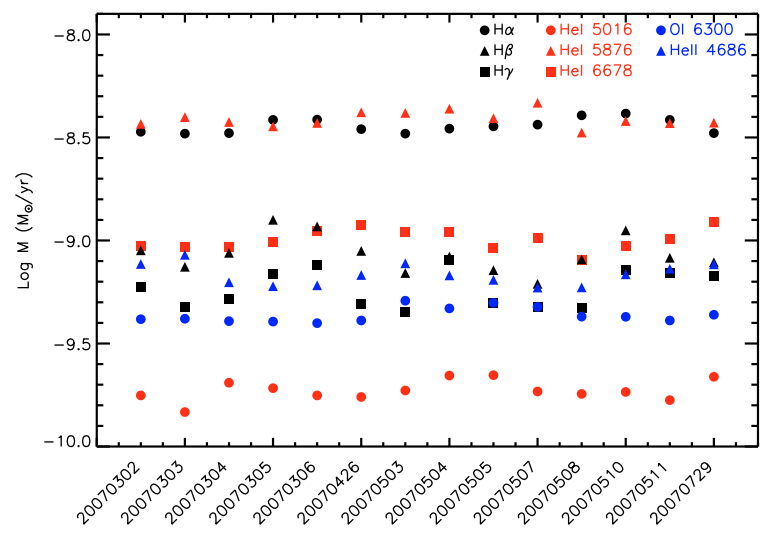

Fig. 3. The variability of TW Hya. The different color/symbols represent different accretion-rate tracers (see upper right corner). Note the $\mathrm{X}$-axis timescale is not linear. The variability in accretion rate is observed to be small in comparison to the range of accretion rates calculated from the different tracers.

in their analysis. Rucinsky et al. (2008) analysed two datasets - from 2007 and 2008 - and found a photometric variability of $\Delta V<0.5 \mathrm{mag}$ on timescales of $\sim 3.5$ days in the 2007 data. However, this was no longer observable in the 2008 data, which showed variability (again $\Delta V<0.5 \mathrm{mag}$ ) on timescales of 29 days possibly owing to the orbital decay of accreting gas. In addition, they find "spikes" in the accretion rate lasting only a fraction of a day. They conclude that whilst accretion contributes to this variability, it is not the sole cause.

Our optical and X-ray observations are not coeval, so it is important to study the variability of these stars due to accretion to determine whether their variability will limit our ability to compare mass accretion rates derived from non-coeval observations. We find that TW Hya, a pole-on star, has a variability within our errors. Furthermore, all the sources in this sample have inclinations $\$ 45^{\circ}$, with TW Hya, Hen 3-600, RU Lup, and T Tau all having inclinations much closer to pole-on, meaning any accretion hotspots would be visible all or most of the time, limiting variability due to observability of the hotspot. Whilst we cannot rule out time-dependent mass-accretion rates for the stars other than TW Hya in our sample, we measure a small variability in our TW Hya data, and even the larger variabilities suggested in the literature fall within our errors.

\subsection{Comparison of optical/ $x$-ray derived accretion rates}

We compare the optically derived mass-accretion rates $\left\langle\dot{M}_{\text {Opt }}\right\rangle$ to the X-ray derived mass-accretion rates $\dot{M}_{\mathrm{X} \text {-ray }}$ in Fig. 4 . The most striking result is that $\dot{M}_{\mathrm{X} \text {-ray }}$ for our sample ranges within a factor of $\approx 2$ around $2 \times 10^{-10} M_{\odot} \mathrm{yr}^{-1}$ (see Fig. 4 and Table 6 ), despite the range of $\left\langle\dot{M}_{\text {Opt }}\right\rangle$ spanning almost three orders of magnitude. In addition, as suggested by other studies in the literature, the Xray mass-accretion rates are always lower than the corresponding optical mass-accretion rates with the discrepancy increasing for increasing $\left\langle\dot{M}_{\text {Opt }}\right\rangle$.

The above results can be interpreted in the light of the findings of Sacco et al. (2010), who used a detailed hydrodynamic model to investigate the observability of accretion shocks in $\mathrm{X}$-rays in a wide range of physical conditions of the accretion stream. They considered the absorption from the optically thick plasma of the stellar chromosphere on the X-ray emission arising from the shock-heated material. They found that the absorption strongly depends on the accretion stream properties and influences the observability of the post-shock accreting plasma in the X-ray band. In particular, observable X-ray emission from accretion shocks is expected in streams with densities in the range $10^{11} \lesssim n_{\mathrm{e}} \lesssim 10^{12} \mathrm{~cm}^{-3}$ and velocities in the range $400 \lesssim v \lesssim 600 \mathrm{~km} \mathrm{~s}^{-1}$. Denser streams produce post-shock zones that are rather narrow and deeply rooted in the chromosphere, causing their X-ray emission to be strongly absorbed. Streams with velocities below $400 \mathrm{~km} \mathrm{~s}^{-1}$ produce shocked plasma with temperatures around $1 \mathrm{MK}$ or even lower and are therefore, hardly observable in X-rays. The obvious consequence is that, whilst accretion shocks are almost always observable in the optical band, only a small fraction of them are detected in X-rays, leading in general to an underestimate of the X-ray derived massaccretion rate and, therefore, to the observed discrepancy with the optically derived mass-accretion rates (see also discussion in Orlando et al. 2010; Sacco et al. 2010).

We note that the above argument is applicable even if only one stream is present on the star. For typical densities and velocities of accretion streams and stellar magnetic field strengths (as derived from observations of CTTSs; Johns-Krull 2007), the plasma $\beta$ (i.e. the ratio of gas pressure to magnetic pressure) is in general, expected to be $\ll 1$ (see Sacco et al. 2010). In these conditions, an accretion stream is a bundle of fibrils each independent of the others because of the strong magnetic field, which prevents mass and energy exchange across magnetic field lines (e.g. Orlando et al. 2010; Sacco et al. 2010). If the stream is structured in density (as suggested, for instance, by 3D MHD models of the star-disk system carried out by Romanova et al. 2004), only fibrils with density and velocity in the range of observability defined by Sacco et al. (2010) are expected to produce detectable X-rays, unless they are located close to the centre of the stream cross-section, where they undergo strong absorption from the accretion column itself.

Interestingly, Hen 3-600 is the lowest mass star in our sample, for which there is close agreement between the optically and $\mathrm{X}$-ray derived mass-accretion rates, implying that it has very few high density streams (or fibrils) - hardly detectable in X-rays or only low density accretion streams (fibrils) - observable in X-rays - meaning almost everything is seen in the X-ray, as well as in the optical. At the other end of the scale, RU Lup might instead have a much larger number of high density streams (fibrils).

If, on the one hand, the discrepancy between optical and $\mathrm{X}$-ray derived mass-accretion rates could be easily explained in the framework of accretion shock models as discussed above, on the other hand, it is more puzzling that $\dot{M}_{\mathrm{X} \text {-ray }}$ is almost the same for all the stars in our sample. This result is even more surprising given that the determination of $\dot{M}_{\mathrm{X} \text {-ray }}$ assumes some fixed parameters (e.g. the distance, mass, and radius of the star, the abundance of the emitting plasma, and the temperature of the post-shock region) whose uncertainties are unknown and therefore not included in the errors in $\dot{M}_{\mathrm{X} \text {-ray }}$. Taking into account all these uncertainties, one would expect to find a scatter in the values of $\dot{M}_{\mathrm{X} \text {-ray }}$ possibly larger than one order of magnitude. In contrast, the evidence is that the observed scatter is within a factor $\approx 2$ and can be explained only if the (unknown) uncertainties in the fixed parameters used in our calculations are smaller than expected.

As for the almost constant value of $\dot{M}_{\mathrm{X} \text {-ray }}$, we note that its estimate relies on the flux of the O VII resonance line and is based on the assumption of optically thin plasma. However, it can be inferred that the optical depth of the strongest emission lines produced by the plasma located in the post-shock region is 

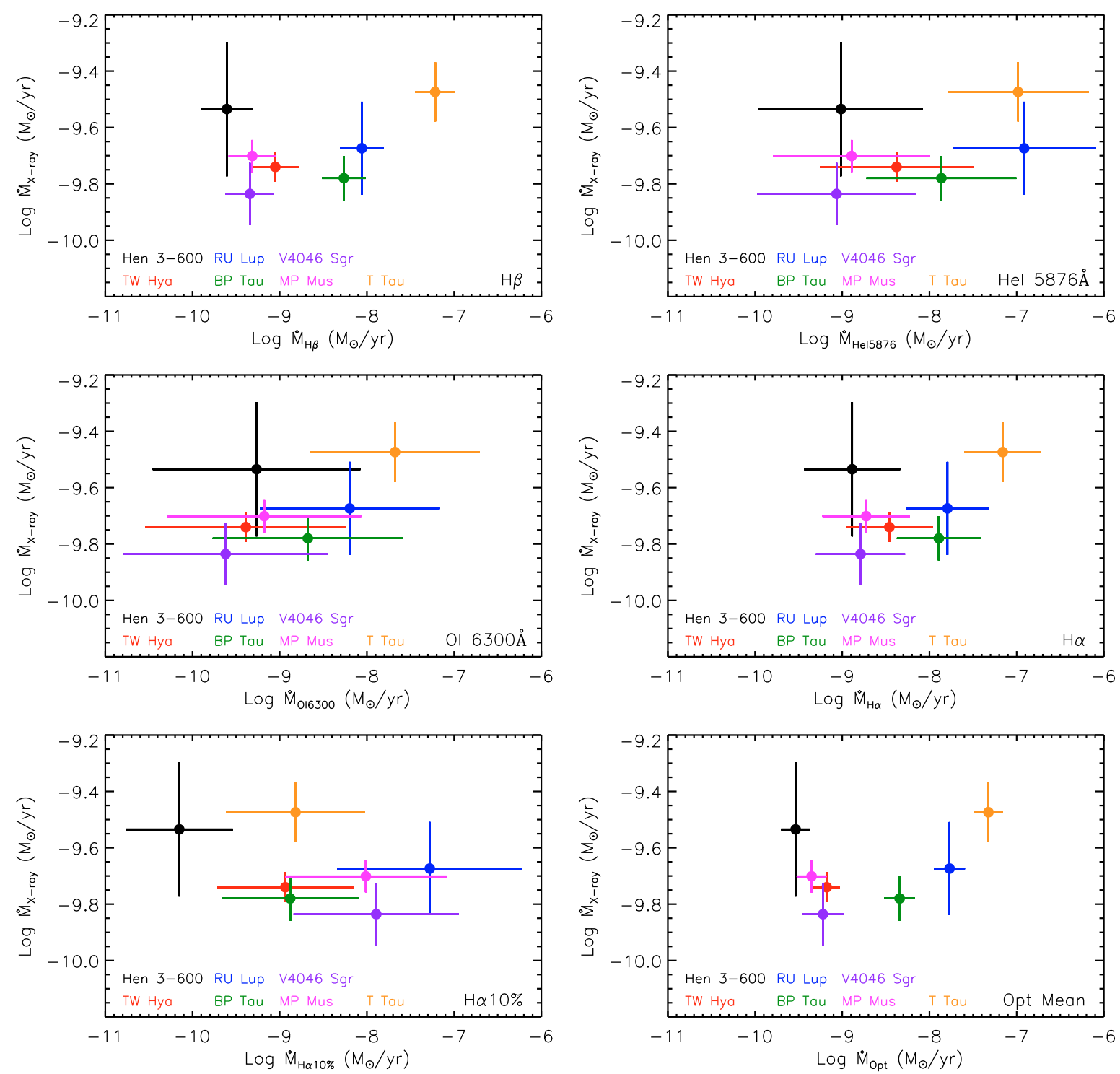

Fig. 4. Plots of the optically derived mass-accretion rates versus the X-ray derived mass-accretion rates. Each panel shows the optical accretion rate derived from a different tracer, indicated in the lower right corner of the plot. The CTTSs are colour coded (see lower left corner of each panel). We plot only those cases for which we have measured the mass-accretion rate for all the stars in our sample (see Table 5). The bottom right panel shows a plot of the optical mean mass-accretion rate plotted against the X-ray accretion rate. There is a very small range, a factor $\approx 2$, in $\mathrm{X}$-ray accretion rate calculated for the entire sample, whereas the optical mass-accretion rates span a range of $\sim 3$ orders of magnitude.

non-negligible. For a post-shock region of dimension $\sim 10^{9} \mathrm{~cm}$, filled with plasma at $T=2 \mathrm{MK}$ and $n_{\mathrm{e}}=10^{11} \mathrm{~cm}^{-3}$, the optical depth of the O VII resonance line is $\sim 10$. Supporting this scenario is some evidence of optical depth effects (i.e. the emitting plasma is not totally optically thin) detected in the X-ray spectrum of MP Mus (Argiroffi et al. 2009), implying that the O VII flux and the derived $\dot{M}_{\mathrm{X} \text {-ray }}$ might be underestimated.

The optical depth effects can be addressed by comparing mass-accretion rates measured from the resonance line of the $\mathrm{O}$ VII triplet with the accretion rate measured using the flux of the intercombination plus the flux of the forbidden line of the same triplet, which are less affected by optical depth effects. We performed this comparison in the case of TW Hya and MP Mus. We found no significant differences in the former case, while the $\dot{M}_{\text {X-ray }}$ of MP Mus measured from the intercombination plus the forbidden line is twice as large as the $\dot{M}_{\mathrm{X} \text {-ray }}$ measured from the resonance line. The lack of high signal-to-noise ratio $(\mathrm{S} / \mathrm{N})$ spectra does not allow us to investigate this in greater detail using this approach for the other stars in our sample. However, this preliminary result suggests that the absorption from the stellar atmosphere is the main cause of the discrepancy between the optical and the $\mathrm{X}$-ray mass accretion rates.

Brickhouse et al. (2010) analysed X-ray spectroscopic data of TW Hya with high $\mathrm{S} / \mathrm{N}$. This kind of analysis is limited to TW Hya, which is the brightest CTTS in the X-ray and has been observed with Chandra for $\sim 500 \mathrm{ks}$. These authors found that the flux and the densities measured from the Ne IX triplet agree with the accretion shock parameters derived from optical emission lines, while the density and flux derived from the O VII triplet disagree. They proposed therefore a model of "accretion-fed corona" in which the X-ray emission originates from three plasma components: a hot $\left(T_{\mathrm{e}} \approx 10 \mathrm{MK}\right)$ corona, a high density $\left(n_{\mathrm{e}} \approx 6.0 \times 10^{12} \mathrm{~cm}^{-3}, T_{\mathrm{e}} \approx 3.0 \mathrm{MK}\right)$ postshock region close to the shock front, and a cold less dense 
$\left(n_{\mathrm{e}} \approx 2 \times 10^{11} \mathrm{~cm}^{-3}, T_{\mathrm{e}} \approx 2.0 \mathrm{MK}\right)$ post-shock cooling region, with 300 times more volume and 30 times more mass than that of the post shock region itself. On the other hand, we note that Sacco et al. (2010) illustrated that, if accretion streams are inhomogeneous and the chromospheric absorption is efficient, their hydrodynamic model of accretion shock predicts that different He-like triplets measure different densities of the X-ray emitting plasma. This is explained because the effect of absorption increases with wavelength and, as a consequence, the Ne IX (13.45 $\AA$ ) emission is less absorbed than O VII (21.60 ̊) emission. In particular, Sacco et al. (2010) showed that, in an inhomogeneous stream affected by chromospheric absorption, the average density measured with the Ne IX triplet is, in general, higher than that measured with O VII triplet, although Ne IX lines form at higher temperatures than O VII lines (as noted by Brickhouse et al. 2010). Unfortunately, owing to the lack of high signal-to-noise ratio $(\mathrm{S} / \mathrm{N})$ data covering the Ne IX triplet, we are unable to test this model for the other stars of our sample. Further long-exposure X-ray spectroscopic observations of the stars in our sample are required to test this model as well as optical depth effects on the estimate of $\dot{M}_{\text {X-ray }}$.

If the results presented in this paper are confirmed, we suggest here that the optical depth effects in the OVII resonance line may explain the almost constant $\dot{M}_{\mathrm{X} \text {-ray }}$ derived in our sample if the effect is larger in stars with larger optically derived mass-accretion rates. Finally, we note that our analysis does not allow us to conclude that there is no relation between $\left\langle\dot{M}_{\text {Opt }}\right\rangle$ and $\dot{M}_{\mathrm{X} \text {-ray }}$. From our analysis, we can just conclude that the relation, if it exists, is masked by the (small) scatter in the values of $\dot{M}_{\mathrm{X}-\text { ray }}$. In this respect, we note that the lower right panel in Fig. 4 seems to suggest that $\dot{M}_{\mathrm{X} \text {-ray }}$ is slightly higher for higher $\left\langle\dot{M}_{\text {Opt }}\right\rangle$ if we consider that the value of $\dot{M}_{\text {X-ray }}$ for Hen 3-600 has the largest error in the sample.

\section{Conclusions}

We have carried out the first homogeneous comparison of optically and X-ray derived mass-accretion rates for a sample of CTTSs. We compare the different optical tracers to each other, and to the X-ray derived accretion rates. We have also analysed the variability of the CTTS TW Hya. Our findings lead to the following conclusions:

1. The mass-accretion rates derived from the different optical tracers agree within the errors for each source, albeit with a large spread of typically $\approx 1$ order of magnitude (see Fig. 1).

2. The $\mathrm{H} \alpha 10 \%$ full width, whilst known as a good indicator of accretion, is not good at measuring mass accretion rates.

3. For the CTTS TW Hya (the only source within the sample for which a time analysis was possible), we find little variation in the mass-accretion rates (for each emission-line tracer) over a period of five months (see Fig. 3). The variability is much smaller than the range of accretion rates derived from different accretion tracers.

4. The X-ray mass-accretion rates are always smaller than the optically derived mass-accretion rates for all sources within the sample (see Fig. 4). This can be explained if the accretion streams are inhomogeneous and/or multiple streams with different densities are present simultaneously. In these cases, Sacco et al. (2010) have shown that the chromospheric absorption triggers a selection of the X-ray emitting shocks, absorbing preferentially the X-ray emission from high density plasma components. As a result, only the low density plasma component of the post-shock plasma can be observed in the X-ray band, leading to a systematic underestimate of the mass-accretion rate.

5. We find that the $\mathrm{X}$-ray derived mass-accretion rate varies within a factor of $\approx 2$ around $2 \times 10^{-10} M_{\odot} \mathrm{yr}^{-1}$ (see Fig. 4), despite the range of optical mass-accretion rates spanning almost three orders of magnitude. Some evidence of nonnegligible optical depth of emission lines produced by postshock accreting plasma (e.g. Argiroffi et al. 2009) may explain the almost constant $\dot{M}_{\mathrm{X} \text {-ray }}$ if the effect is larger in stars with higher optical mass-accretion rates. This issue deserves further investigation in future studies to assess the severity of optical depth effects on the estimate of mass-accretion rates in the X-ray band.

Acknowledgements. Based on observations made with ESO Telescopes at the La Silla or Paranal Observatories under programme ID $\langle 069 . C-0481\rangle,\langle 073 . C-$ $0355\rangle,\langle 075 . C-0292\rangle,\langle 077 . D-0478\rangle,\langle 078 . A-9059\rangle,\langle 079 . A-9006\rangle$, $\langle 079 . A-9007\rangle$ and $\langle 079 . A-9017\rangle$. Based on observations made with the Italian Telescopio Nazionale Galileo (TNG) operated on the island of La Palma by the Fundación Galileo Galilei of the INAF (Istituto Nazionale di Astrofisica) at the Spanish Observatorio del Roque de los Muchachos of the Instituto de Astrofisica de Canarias. This work was supported by the EU Marie Curie Transfer of Knowledge program PHOENIX under contract No. MTKD-CT2005-029768 and in part by Agenzia Spaziale Italiana under contract No. ASIINAF I/088/06/0.

\section{References}

Akeson, R. L., Koerner, D. W., \& Jensen, E. L. N. 1998, ApJ, 505, 358 Alencar, S. H. P., \& Batalha, C. 2002, ApJ, 571, 378

Argiroffi, C., Maggio, A., \& Peres, G. 2007, A\&A, 465, L5

Argiroffi, C., Maggio, A., Peres, G., et al. 2009, A\&A, 507, 939

Batalha, C. C., Quast, G. R., Torres, C. A. O., et al. 1998, A\&AS, 128, 561

Bertout, C., Basri, G., \& Bouvier, J. 1988, ApJ, 330, 350

Brickhouse, N. S., Cranmer, S. R., Dupree, A. K., et al. 2010, ApJ, 710, 1835

Calvet, N., \& Gullbring, E. 1998, ApJ, 509, 802

Carpenter, J. M., Wolf, S., Schreyer, K., Launhardt, R., \& Henning, T. 2005, AJ, 129,1049

Comerón, F. 2008, Handbook of Star Forming Regions Volume II., ed. B. Reipurth, 295

Cortes, S. R., Meyer, M. R., Carpenter, J. M., et al. 2009, ApJ, 697, 1305

Costa, V. M.., Lago, M. T. V. T., Norci, L., \& Meurs, E. J. A. 2000, A\&A, 354, 621

Dahm, S. E., 2008, AJ, 136, 512

Donati, J.-F., Jardine, M. M., Gregory, S. G., et al., 2007, MNRAS, 380, 1297

Donati, J.-F., Jardine, M. M., Gregory, S. G., et al. 2008, MNRAS, 386, 1234

Feigelson, E. D., \& DeCampli, W. M. 1981, ApJ, 243, L89

Feigelson, E. D., \& Montmerle, T. 1999, ARA\&A, 37, 363

Geoffray, H., \& Monin, J.-L. 2001, A\&A, 369, 239

Grankin, K. N., Melnikov, S. Yu., Bouvier, J., Herbst, W., \& Shevchenko, V. S. 2007, A\&A, 461, 183

Güdel, M., \& Telleschi, A. 2007, A\&A, 474, 25

Güdel, M., Skinner, S. L., Mel'nikov, S. Yu., et al. 2007 A\&A, 468, 529

Gullbring, E., 1994, A\&A, 287, 131

Gullbring, E., Hartmann, L., Briceno, C., \& Calvet, N. 1998, ApJ, 492, 323

Günther, H. M., Liefke, C., Schmitt, J. H. M. M., Robrade, J., \& Ness, J.-U. 2006 A\&A, 459, L29

Günther, H. M., Schmitt, J. H. M. M., Robrade, J., et al., 2007, A\&A, 466, 111

Herczeg, G. J., \& Hillenbrand, L. A. 2008, ApJ, 681, 594

Herczeg, G. J., Walter, F. M., Linsky, J. L., et al. 2005, AJ, 129, 2777

Huenemoerder, D. P., Kastner, J. H., Testa, P., Schulz, N. S., \& Weintraub, D. A. 2007, ApJ, 671, 592

Hughes, J., Hartigan, P., \& Clampitt, L. 1993, AJ, 105, 571

Hutchinson, M. G., Evans, A., Winkler, H., \& Spencer-Jones, J. 1990, A\&A, 234, 230

Jayawardhana, R., Hartmann, L., Fazio, G., et al. 1999, ApJ, 520, L41

Johns-Krull, C. M. 2007, ApJ, 664, 975

Johnson, H. L. 1966, ARA\&A, 4, 193

Kastner, J. H., Zuckerman, B., Weintraub, D. A., \& Forville, T. 1997, Science, 277,67

Kastner, J. H., Huenemoerder, D. P., Schulz, N. S., Canizares, C. R., \& Weintraub, D. A. 2002, ApJ, 567, 434

Kastner, J. H., Zuckerman, B., Hily-Blant, P., \& Forveille, T. 2008, A\&A, 492, 469 
R. L. Curran et al.: Multiwavelength diagnostics of accretion in CTTSs

Kenyon, S. J., \& Hartmann, L. 1995, ApJS, 101, 117

Kenyon, S. J., Gómez, M., \& Whitney, B. A. 2008, Handbook of Star Forming Regions Volume I., ed. B. Reipurth, 405

Koldoba, A. V., Ustyugova, G. V., Romanova, M. M., \& Lovelace, R. V. E. 2008, MNRAS, 388, 357

Lamzin, S. A. 1998, Astron. Rep., 42, 322

Lasker, B. M., Lattanzi, M. G., McLean, B. J., et al. 2008, AJ, 136, 735

Lombardi, M., Lada, C. J., \& Alves, J. 2008, A\&A, 480, 785

Mamajek, E. E., Meyer, M. R., \& Liebert, J. W. 2002, ApJ, 124, 1670

Mekkaden, M. V. 1998, A\&A, 340, 135

Mitchell, R. I., \& Johnson, H. L. 1969, Commun. Lunar Planet. Lab, 8, 1

Mohanty, S., Jayawardhana, R., \& Barrado y Navascués, D. 2003, ApJ, 593, 109

Mohanty, S., Jayawardhana, R., \& Basri, G. 2005, ApJ, 626, 498

Muzerolle, J., Hartmann, L., \& Calvet, N. 1998, AJ, 116, 455

Muzerolle, J., Calvet, N., Briceño, C., Hartmann, L., \& Hillenbrand, L. 2000, ApJ, 535, L47

Natta, A., Testi, L., Muzerolle, J., et al. 2004, A\&A, 424, 603

Natta, A., Testi, L., \& Randich, S. 2006, A\&A, 452, 245

Orlando, S., Sacco, G. G., Argiroffi, C., et al. 2010, A\&A, 510, A71

Pickles, A. J. 1985, ApJS, 59, 33

Quast, G. R., Torres, C. A. O., de La Rezza, R., da Silva, L., \& Mayor, M. 2000, in IAU Symp., 28P

Qi, C., Ho, P. T. P., Wilner, D. J., et al. 2004, ApJ, 616, L11

Raassen, A. J. J. 2009, A\&A, 505, 755

Robrade, J., \& Schmitt, J. H. M. M. 2006, A\&A, 449, 737
Robrade, J., \& Schmitt, J. H. M. M. 2007, A\&A, 473, 229

Romanova, M. M., Ustyugova, G. V., Koldoba, A. V., \& Lovelace, R. V. E. 2004, ApJ, 610, 920

Rucinsky, S. M., Matthews, J. M., Kuschnig, R., et al. 2008, MNRAS, 391, 1913

Sacco, G. G., Argiroffi, C., Orlando, S., et al. 2008, A\&A, 491, L17

Sacco, G. G., Orlando, S., Argiroffi, C., et al. 2010, A\&A, 522, A55

Schmitt, J. H. M. M., Robrade, J., Ness, J.-U., Favata, F., \& Stelzer, B. 2005, A\&A, 432, L35

Scholz, A., Jayawardhana, R., \& Brandeker, A. 2005, ApJ, 629, L41

Silverstone, M. D., Meyer, M. R., Mamajek, E. E., et al. 2006, ApJ, 639, 1138

Solf, J., \& Böhm, K.-H. 1999 ApJ, 523, 709

Song, I., Zuckerman, B., \& Bessell, M. S. 2003, ApJ, 599, 342

Stelzer, B., \& Schmitt, J. H. M. M. 2004, A\&A, 418, 687

Stempels, H. C., \& Piskunov, N. 2002 A\&A, 391, 595

Stempels, H. C., \& Gahm, G. F. 2004, A\&A, 421, 1159

Stempels, H. C., Gahm, G. F., \& Petrov, P. P. 2007, A\&A, 461, 253

Telleschi, A., Güdel, M., Briggs, K. R., Audard, M., \& Scelsi, L. 2007, A\&A, 468,443

Torres, C. A., da Silva, L., Quast, G. R., de la Reza, R., \& Jilinski, E. 2000, AJ, 120,1410

Torres, C. A. O., Quast G. R., Melo, C. H. F., \& Sterzik, M. F. 2008, Handbook of Star Forming Regions Volume II., ed. B. Reipurth, 757

Wichmann, R., Bastien, U., Krautter, J., et al. 1998, MNRAS, 301, L39

Wilking, B. A., Meyer, M. R., Robinson, J. G., \& Greene, T. P. 2005, AJ, 130, 1733 